Motion Analysis in AMC

Rodriguez, Bickley et al.

\title{
Perspectives on Gait and Motion Analysis in the Management of Youth with Arthrogryposis Multiplex Congenita
}

Luisa Maria Rodriguez ${ }^{1}$, Christina Bickley ${ }^{2,3}$, Stephanie Russo ${ }^{4}$, Douglas Barnes ${ }^{2}$

Marianne Gagnon ${ }^{5,6}$, Reggie Hamdy ${ }^{5,6}$, Louis-Nicolas Veilleux ${ }^{5,6}$

${ }^{1}$ Motion Analysis Center, Shriners Hospitals for Children - Philadelphia

${ }^{2}$ Motion Analysis Center, Shriners Hospitals for Children - Houston

3 Texas Woman's University, School of Physical Therapy - Houston

${ }^{4}$ Department of Orthopaedic Surgery, University of Pittsburgh Medical Center - Hamot

${ }^{5}$ Motion Analysis Center, Shriners Hospitals for Children-Canada

${ }^{6}$ Departement of Surgery, Faculty of Medicine, McGill University

Running Title: Motion Analysis in AMC

Corresponding Author: Louis-Nicolas Veilleux

Motion Analysis Center

Shriners Hospital for Children-Canada

1003 Decarie Blvd.

Montréal, Quebec, Canada H4A 0A9 


\begin{abstract}
Arthrogryposis multiplex congenita (AMC) is a rare congenital musculoskeletal disease ( 1 in 4300) characterized by joint contractures present in two or more body areas. Some studies have shown that people with AMC have reduced functional ability and various degrees of gait abnormalities. Different types of surgery or assistive technical aids can be prescribed to improve their autonomy and quality of life. Quantified motion analysis (QMA) for upper limbs and trunk and quantified gait analysis for lower limbs (QGA) are the systematic evaluations of human movement. The goal of this manuscript is to identify potential avenues for the implementation of QMA and QGA in the management and follow-up of youth with AMC. Applications for children with AMC include quantifying motion or gait and monitoring function during development, treatment planning, and outcomes assessment. Many challenges are remaining to fill the gaps in the literature regarding the role of QMA and QGA in the management of youth with AMC. Additional efforts from the clinical research community are needed to improve the contribution of motion analysis in the selection of surgical and/or non-surgical therapeutic approaches and documentation of the associated improvement in the functional outcomes for the AMC population.
\end{abstract}

Key words: arthrogryposis multiplex congenita, gait analysis, motion analysis, treatment planning, treatment outcomes 
Arthrogryposis multiplex congenita (AMC) is a generic term that encompasses many different types of multiple congenital contractures present at birth and affecting between 1 in 4300 to 5100 live births (Darin, Kimber, Kroksmark, \& Tulinius, 2002; Lowry, Sibbald, Bedard, \& Hall, 2010; Staheli, Hall, Jaffe, \& Paholke, 1999). Amyoplasia is the most common type of AMC with an incidence of 1 in 10000 live births followed by distal arthrogryposis (Staheli et al., 1999). Many factors can cause AMC with the most frequent potential explanation being the lack of fetal movement (Gordon, 1998; Kalampokas, Kalampokas, Sofoudis, Deligeoroglou, \& Botsis, 2012). Clinical diagnosis of AMC is provided when joint contractures are present in two or more body areas. In addition to these contractures, individuals with AMC can have low bone mass, bone deformities and pain (Hamdy \& Dahan-Oliel, 2016; Kimber, Tajsharghi, Kroksmark, Oldfors, \& Tulinius, 2012; Spencer, Bowen, Caputo, Green, \& Lawrence, 2010; Veilleux \& Rauch, 2017). All body joints can be affected, the most frequent being foot, hip, elbow and knee (Gordon, 1998). Some studies have shown that people with AMC have reduced functional ability (Bharucha, Pandya, \& Dastur, 1972; Nouraei, Sawatzky, MacGillivray, \& Hall, 2017; Spencer et al., 2010) and various degrees of gait abnormalities depending on the severity of the diagnosis and types of technical walking aids (Amor, Spaeth, Chafey, \& Gogola, 2011; Eriksson, Bartonek, Ponten, \& Gutierrez-Farewik, 2015; Eriksson, Gutierrez-Farewik, Brostrom, \& Bartonek, 2010).

There are multiple approaches to the management of AMC youth walking pattern. One such approach is physical and occupational therapy. These therapeutic interventions address body function with the aim to maximize functional independence through increasing range of motion (ROM), preventing recurrence of contractures, and/or addressing complaints of pain and muscle weakness (Borowski et al., 2008; Chomiak, Dungl, \& Vcelak, 2014; Doi, Arakawa, Hattori, \& Baliarsing, 2011; Toydemir \& Bamshad, 2009). Over the course of their lives, 94\% of AMC patients will actively participate in physical therapy and $79 \%$ in occupational therapy (Sells, Jaffe, \& Hall, 1996). Casting and splinting are also frequently prescribed in the management of AMC aiming to improve joint ROM and reduce the need for surgery (Graydon \& Eastwood, 2014). Another important treatment approach is surgery. Individuals with AMC undergo an average of 5.8 surgeries throughout their life (Obeidat, Audat, \& Khriesat, 2012; Sells et al., 1996). The goal of many of these surgeries is to allow and/or improve daily activities as self-care, sitting, standing and walking ability and participation realigning upper and lower limbs to optimize ROM and motor function.

Quantified gait analysis for lower limbs (QGA) and quantified motion analysis for upper limbs and trunk (QMA) are the systematic evaluations of human movement. These evaluations include but are not restricted to walking, through the participation of experienced evaluators and supported by multiple cutting-edge technologies. QGA and QMA occur in a Motion Analysis Laboratory (MAL) or Center (MAC) and allows evaluation of limb range of motion, mechanics and muscle activity. QGA has been around for at least 30 years, but only recently has literature emerged to support its role in the management and follow-up of pediatric disorders. To date, cerebral palsy (CP) is the most frequently studied pediatric population in the field of motion analysis. In that regard, a recent randomized-controlled trial (RCT) comparing two pediatric $\mathrm{CP}$ patients' groups: one group underwent a QGA prior to surgical interventions whereas the other group did not. The goal of the study was to determine whether post-surgical outcomes would be better for those patients who benefited from a QGA report guiding pre-surgical decision. Results 
showed significant difference in the pain and physical function scores of the Pediatric Outcomes Data Collection Instrument (PODCI) and change in health in the Child Health Questionnaire (CHQ) compared to the control group (Wren et al., 2013). This suggests that QGA reports improved pre-surgical decision which in turns improved functional outcomes. Despite some success, the results of this RCT were mitigated by the fact that only a small proportion (42\%) of QGA-based recommendations were followed by orthopedic surgeons. Two conclusions can be drawn from these results: (1) Pre-operative quantified gait analysis can lead to improved postoperative functional outcomes of the upper and lower limbs and (2) additional RCTs are needed to support the benefits of QGA in the management and follow-up of pediatric musculoskeletal disorders to inform orthopedic surgeons and other clinicians in their decision-making process.

Much of the pediatric QGA literature is on ambulatory children and adolescents with CP undergoing multi-level orthopedic surgery to improve ambulation. At present, it is still unclear whether QMA can complement the current standard of care armamentarium for young patients with AMC. The current literature published on gait analysis in AMC is scarce and only includes descriptive studies. Given that most therapeutic interventions in AMC are directed towards improving the individual's function and independence, QGA and QMA could be implemented to assess the success of these interventions and potentially improve selection of appropriate surgical and non-surgical interventions. The goal of the present manuscript is to identify the potential avenues to implement QMA and QGA in the management and follow-up of upper and lower limb motor function in youth with AMC.

\section{Quantified motion analysis for the management and follow-up of upper-limb motor function in AMC}

In the AMC population, the typical presentation of the shoulders is adduction and internal rotation (Bennett, Hansen, Granberry, \& Cain, 1985; Ezaki \& Carter, 2004). Weak deltoid function leads to inability to abduct the upper limb against gravity. The elbows frequently present with an extension contracture, wrists are in flexion and ulnar deviation, thumbs are adducted in the palm, and fingers are partially flexed and rigid (Bennett et al., 1985; Ezaki \& Carter, 2004). Due to the atypical positioning of the upper limb, difficulties in activity of daily life (ADL's) such as toileting, self-feeding, dressing up, and dental and facial care, are seen in children with arthrogryposis.

The aim of upper limb treatment is to improve quality of life by facilitating functional independence. Conservative (i.e. nonoperative) therapeutic interventions include stretching, splinting, casting, and occupational and physical therapy (Palmer, MacEwen, Bowen, \& Mathews, 1985). If functional limitations persist after conservative therapeutic treatment, then surgical therapeutic intervention may be pursued. In brief, common surgical interventions for children with AMC include: (1) external rotation humeral osteotomies to improve position of the limb, elbow releases to improve ROM and the ability to self-feed and; (2) carpal wedge and thumb reorientation osteotomies as well as webspace deepening to improve pinch, object manipulation, and object acquisition (Ezaki \& Carter, 2004; Foy, Mills, Wheeler, Ezaki, \& Oishi, 2013; Zlotolow, 2017; Zlotolow \& Kozin, 2012; Zlotolow \& Tiedeken, 2014). A detailed description on the orthopedic management of the upper limb can be found in another section of

42 this special edition (Oishi et al.). 
Upper limb surgical planning can be assisted with quantified motion analysis (QMA). Motion capture technology is an essential component of QMA and is an evolving tool for use in the upper limb. Applications for children with AMC include quantifying motion and monitoring function during development, surgical planning, and outcomes assessment. Motion capture can be utilized to obtain objective measures of joint ROM during prescribed and functional tasks such as drinking from a glass, manipulating objects in their hands, picking up a telephone or reaching an object on a shelf. It could also be used to quantify compensatory strategies such as spinal movements. Additionally, capturing functional workspace measures of the upper limb, or specific joints such as the thumb carpometacarpal joint, are particularly relevant for this population. Data obtained from motion capture assessments may be utilized to assist in determining the optimal degrees of rotation for external humeral rotation osteotomies. Similarly, motion capture assessments can augment indications for triceps lengthening/posterior elbow release, carpal wedge osteotomy, and thumb reorientation osteotomies (Curran, Bagley, SisonWilliamson, \& James, 2019). Outcomes following surgical and nonsurgical interventions may be measured, as well. Serial motion capture assessments can determine if successful outcomes are maintained over time as well as add to the longitudinal knowledge of a patient population.

One example of the use of motion analysis to evaluate outcomes in children with AMC is through the documentation of functional changes in children who have been prescribed upper limb orthoses. For instance, custom fabricated wrist-hand orthoses (WHO) for use on assistive devices such as walkers or loft strand crutches have been fabricated to improve the child's ability to manipulate the assistive device during ambulation (see figures 1-2). The exact components of an upper limb QMA would depend on the purpose and design of the upper limb orthosis. Videotaping, patient and parent subjective reports, and functional outcome measures have been employed to evaluate the effect of WHOs in this patient population. Evaluation of the effect of assistive devices with custom WHOs on function is an example of the multi-faceted utility of the QMA to meet the evaluation and assessment needs of patients with AMC.

Although the clinical use of QMA is an important adjunct that offers objective data to support and evaluate upper limb treatment decision making, there is limited research available. To date, there is one published study using QMA of the upper limb in AMC to evaluate upper limb orthotic intervention. This case study by Haumont et al. (2011) involved one patient with AMC, and two patients with spinal muscular atrophy (SMA). All three subjects underwent QMA to test the Wilmington Robotic Exoskeleton (WREX), an upper limb orthosis. The patients were tested with and without the device. The authors purport that using the WREX improved upper limb mobility by allowing increased ranges of motion of the shoulder and elbow of the tested patients. While motion analysis data were made available for both SMA patients, none were reported for the AMC patient limiting the conclusions related to motion analysis in the management of AMC for this upper limb device.

\section{Quantified gait analysis for the management and follow-up of lower limbs motor function in AMC}

As currently used in pediatric motion analysis centers (MACs) across the world, quantified gait analysis aims at evaluating the efficiency of lower limb interventions such as physical therapy, orthotics and surgery. Such analysis informs clinicians for future treatment decisions which in turn leads to improve function and participation of children and adolescents. 
Most of the current literature on this topic is related to youth with CP but this approach could apply to children and adolescents with AMC. As it stands now, only a few studies have been published in which QGA was performed in youth with AMC. These studies either described gait patterns in patients with AMC, such as trunk motion during gait (Bohm, Dussa, Multerer, \& Doderlein, 2013), or evaluated changes in gait outcome parameters following orthotic use (Bartonek, 2015). QGA is generally used clinically in youth with AMC to identify best suited therapeutic interventions, such as orthotics, aiming at facilitating movement, improving motor function, and increasing patient participation.

Orthotics are one type of non-surgical intervention that can have a significant impact on Bosse, 2015). The QGA components (physical exam, video recording of gait in coronal and sagittal planes, pedobarograph, instrumented gait analysis, energy consumption, etc.) that make up an orthotic gait analysis are dependent upon the type and purposes of the device being evaluated. A study from Eriksson compared gait parameters between barefoot and orthotic conditions in youth with AMC. Results indicated improved stride and temporal parameters for patients wearing the orthotics but only in a small number of patients (Eriksson et al., 2010). While the literature supports the use of orthoses to improve the gait of children with AMC to maximize patient function through orthotic intervention (Bartonek, Eriksson, \& GutierrezFarewik, 2007; Eriksson et al., 2015), QGA data are needed to develop optimal orthotic prescription and document the associated benefits of varying designs and materials.

Orthotics optimization can be achieved using different materials and innovative designs. One such innovation is the utilization of Carbon Fiber Knee and Ankle Foot Orthoses (CFKFAOs and CF-AFOs) in youth with AMC (see figure 3). Bartonek et al. (Bartonek et al., 2007) did gait analysis on 12 myelomeningocele (MMC) and four AMC children to compare CF-AFOs and CF-KAFOs to regular thermo-plastic orthoses during gait. They have shown that CFSOs did not give same results in both populations: CF-AFOs and CF-KAFOs enhance gait function in MMC by improving significantly ankle plantar flexion moment, ankle positive work, knee extension moment, and stride length when significant improvement in AMC was seen only for ankle absorption. Gait of children with $\mathrm{CP}$ displaying stiff knee gait has shown increased knee flexion during the swing phase of gait using the CF-AFO (Jagadamma et al., 2009). Based on these observations in $\mathrm{CP}$ and given that children with $\mathrm{AMC}$ also display decreased use of available knee flexion during the swing phase of the gait cycle, this suggests that benefits of CFAFOs to address this gait deviation may also apply to patients with AMC. Current personal observations suggest gait outcome parameters benefits related to the CF-AFO, like improved knee flexion during swing phase and improved ankle and knee motion during the loading response phase of gait. Kinetic trends seen with CF-AFO use appear to improve ankle joint moments during the loading response and improved power generation at the ankle during the terminal stance. Considering the dynamic properties of carbon fiber material, as opposed to the rigidity of thermoplastic, these findings are logical. Common patient and parent subjective data regarding use of CF-AFOs and CF-KAFOs included reports of decreased tripping and falling, improved tolerance to CF-AFOs and CF-KAFOs wear, enhanced standing stability, improved walking ability, increased walking velocity, and better shoes selection options. Despite these encouraging observations, additional studies are needed to assess multiple walking conditions comparing ambulation with CF-AFOs and CF-KAFOs to traditional solid thermoplastic AFOs and KFAOs. 
While the discussion of the benefits of QGA for lower limb management has focused on the role of gait analysis in the optimization of orthoses for gait function in youth with AMC, the principles detailed in the section above could apply to assessing pre-surgical gait analysis and post-surgical patient functional outcomes. Clubfoot deformities, knee contractures and hip dislocations are the most common orthopedic deficits reported in AMC (van Bosse et al., 2017) and are most likely to affect gait patterns in youth with AMC. Surgical management of the lower limbs are frequent in AMC with $76 \%$ of children undergoing foot surgery, $39 \%$ knee surgery and $16 \%$ hip surgery (Bamshad, Van Heest, \& Pleasure, 2009) and QGA could contribute to understanding the efficiency of surgeries in restoring partial function of the lower limbs. Despite some available literature, there is a dearth of data on the potential role of gait analysis in improving pre-surgical planning and in assessing improved gait following surgical or physical interventions, such as in the case of CP. There is therefore a need to develop randomized controlled trials comparing post-surgical outcomes in groups of patients with AMC benefiting, or not, from a pre-surgical gait analysis report.

QGA is not limited to kinematics and kinetics but also provides spatiotemporal gait outcome parameters, energy expenditure data and plantar pressure data. AMC is a complex musculoskeletal disorder and limiting analysis to kinematics and kinetics would miss a fair proportion of functional deficits these children have. For example, a study from Eriksson and colleagues have shown that despite wearing orthoses, patients with AMC still had increased energy expenditure during gait compared to typically developing children. Therefore, having a measure of gait efficiency (i.e., energy expenditure) is an important outcome to account for when orthoses or surgical interventions are planned and done. Pedobarographs are a component of QGA and could be used pre and post-operatively to assess the change in foot deformities (Bowen, Miller, Castagno, Richards, \& Lipton, 1998). Patients with distal AMC will also frequently have talipes equinovarus (clubfoot). This complex foot deformity affects pressure distribution under the foot and despite treatment being efficient in correcting the deformity, some atypical pressure distribution can remain (Jeans, Erdman, \& Karol, 2017). This analysis is already use with foot deformity present in other pediatric disease like with Charcot-Marie-Tooth disease (Erickson et al., 2015).

\section{Discussion and Conclusions}

The literature is beginning to recognize the use of motion analysis for assessment of children with AMC and the value this data can add to guide treatment decisions and measure outcomes in the AMC population. Going forward, motion analysis centers should continue growing and tailoring services offered to this patient population in order to provide quantifiable data to help clinicians discern the most effective treatment interventions. For example, a balance assessment providing a microanalysis of standing center of pressure during a 30 -second data capture could be implemented in clinics to provide practitioners' quantitative data to support anecdotal improvements in balance reported by patients during, or after, treatment intervention. Another service that could improve our understanding of gait and upper limb function in AMC would be the use of a full body motion model that would include detailed information related to movement of the head, arms, and trunk motion in relation to lower limbs gait patterns. This additional motion analysis information may further assist in providing a more thorough evaluation of the benefits of various treatment options that otherwise would go unnoticed due to a lack of quantitative capture of this movement. 
1 Many challenges are remaining to fill the gaps in the literature regarding the role of QMA 2 and QGA in the management of youth with AMC. Despite some literature on the topic, efforts 3 need to increase regarding the role of motion analysis in the selection of surgical and/or non4 surgical therapeutic approaches and documentation of the associated improvement in the 5 functional outcomes for the AMC population. Other challenges, technical or clinical, may arise. 6 For example, recording of the motion data maybe affected by severe muscle contracture and 7 bony deformities which can affect accurate motion analysis marker placement on the patient's 8 anatomical landmark. The anatomical variations seen with the AMC population also 9 complexifies goniometric and anthropometric measurements. Many ambulatory patients will 10 have orthoses that make placement of the motion analysis markers more challenging. The large 11 variability of patient's functional status and the absence of a functional classification, such as 12 GMFCS in CP, makes it challenging to perform grouped analyses for the purposes of outcomes 13 assessment.

14

15 Conflict of Interest: The authors declare no conflict of interest. 
Motion Analysis in AMC

Rodriguez, Bickley et al.

\section{References}

Amor, C. J., Spaeth, M. C., Chafey, D. H., \& Gogola, G. R. (2011). Use of the Pediatric Outcomes Data Collection Instrument to evaluate functional outcomes in arthrogryposis. J Pediatr Orthop, 31(3), 293-296. doi:10.1097/BPO.0b013e31820cad93

Bamshad, M., Van Heest, A. E., \& Pleasure, D. (2009). Arthrogryposis: a review and update. $J$ Bone Joint Surg Am, 91 Suppl 4, 40-46. doi:10.2106/JBJS.I.00281

Bartonek, A. (2015). The use of orthoses and gait analysis in children with AMC. J Child Orthop, 9(6), 437-447. doi:10.1007/s11832-015-0691-7

Bartonek, A., Eriksson, M., \& Gutierrez-Farewik, E. M. (2007). Effects of carbon fibre spring orthoses on gait in ambulatory children with motor disorders and plantarflexor weakness. Dev Med Child Neurol, 49(8), 615-620. doi:10.1111/j.1469-8749.2007.00615.x

Bennett, J. B., Hansen, P. E., Granberry, W. M., \& Cain, T. E. (1985). Surgical management of arthrogryposis in the upper extremity. J Pediatr Orthop, 5(3), 281-286.

Bharucha, E. P., Pandya, S. S., \& Dastur, D. K. (1972). Arthrogryposis multiplex congenita: Part 1: Clinical and electromyographic aspects. J Neurol Neurosurg Psychiatry, 35(4), $425-$ 434.

Bohm, H., Dussa, C. U., Multerer, C., \& Doderlein, L. (2013). Pathological trunk motion during walking in children with amyoplasia: is it caused by muscular weakness or joint contractures? Res Dev Disabil, 34(11), 4286-4292. doi:10.1016/j.ridd.2013.09.020

Borowski, A., Grissom, L., Littleton, A. G., Donohoe, M., King, M., \& Kumar, S. J. (2008). Diagnostic imaging of the knee in children with arthrogryposis and knee extension or hyperextension contracture. J Pediatr Orthop, 28(4), 466-470. doi:10.1097/BPO.0b013e31816c4dd8

Bowen, T. R., Miller, F., Castagno, P., Richards, J., \& Lipton, G. (1998). A method of dynamic foot-pressure measurement for the evaluation of pediatric orthopaedic foot deformities. $J$ Pediatr Orthop, 18(6), 789-793.

Chomiak, J., Dungl, P., \& Vcelak, J. (2014). Reconstruction of elbow flexion in arthrogryposis multiplex congenita type I: results of transfer of pectoralis major muscle with follow-up at skeletal maturity. J Pediatr Orthop, 34(8), 799-807. doi:10.1097/BPO.0000000000000204

Curran, P. F., Bagley, A. M., Sison-Williamson, M., \& James, M. A. (2019). Three-dimensional functional workspace of thumb prehension. Clin Biomech (Bristol, Avon), 63, 63-72. doi:10.1016/j.clinbiomech.2019.02.017

Darin, N., Kimber, E., Kroksmark, A. K., \& Tulinius, M. (2002). Multiple congenital contractures: birth prevalence, etiology, and outcome. J Pediatr, 140(1), 61-67. doi:10.1067/mpd.2002.121148

Doi, K., Arakawa, Y., Hattori, Y., \& Baliarsing, A. S. (2011). Restoration of elbow flexion with functioning free muscle transfer in arthrogryposis: a report of two cases. J Bone Joint Surg Am, 93(18), e105. doi:10.2106/JBJS.J.01846

Erickson, S., Hosseinzadeh, P., Iwinski, H. J., Muchow, R. C., Talwalkar, V. R., Walker, J. L., \& Milbrandt, T. A. (2015). Dynamic pedobarography and radiographic evaluation of surgically treated cavovarus foot deformity in children with Charcot-Marie-Tooth disease. J Pediatr Orthop B, 24(4), 336-340. doi:10.1097/BPB.0000000000000163

Eriksson, M., Bartonek, A., Ponten, E., \& Gutierrez-Farewik, E. M. (2015). Gait dynamics in the wide spectrum of children with arthrogryposis: a descriptive study. BMC Musculoskelet Disord, 16, 384. doi:10.1186/s12891-015-0834-5 
Eriksson, M., Gutierrez-Farewik, E. M., Brostrom, E., \& Bartonek, A. (2010). Gait in children with arthrogryposis multiplex congenita. $J$ Child Orthop, 4(1), 21-31. doi:10.1007/s11832-009-0234-1

Ezaki, M., \& Carter, P. R. (2004). Carpal wedge osteotomy for the arthrogrypotic wrist. Tech Hand Up Extrem Surg, 8(4), 224-228.

Foy, C. A., Mills, J., Wheeler, L., Ezaki, M., \& Oishi, S. N. (2013). Long-term outcome following carpal wedge osteotomy in the arthrogrypotic patient. J Bone Joint Surg Am, 95(20), e150. doi:10.2106/JBJS.L.01122

Gordon, N. (1998). Arthrogryposis multiplex congenita. Brain \& Development, 20(7), 507-511.

Graydon, A. J., \& Eastwood, D. M. (2014). Orthopaedic Management of Arthrogryposis Multiplex Congenita. In G. Bentley (Ed.), European Surgical Orthopaedics and Traumatology (pp. 4627-4644). Berlin, Heidelberg: Springer Berlin Heidelberg.

Hall, J. G., Agranovich, O., Ponten, E., \& van Bosse, H. J. (2015). Summary of the 2nd International Symposium on Arthrogryposis, St. Petersburg, Russia, September 17-19, 2014. Am J Med Genet A, 167(6), 1193-1195. doi:10.1002/ajmg.a.36938

Hamdy, R. C., \& Dahan-Oliel, N. (2016). Arthogryposis. In S. Sabharwal (Ed.), Pediatric Lower Limb Deformities : Principles and Techniques of Management (1st ed. 2016. ed.). Cham: Springer International Publishing. doi:10.1007/978-3-319-17097-8

Haumont, T., Rahman, T., Sample, W., M, M. K., Church, C., Henley, J., \& Jayakumar, S. (2011). Wilmington robotic exoskeleton: a novel device to maintain arm improvement in muscular disease. J Pediatr Orthop, 31(5), e44-49. doi:10.1097/BPO.0b013e31821f50b5

Jagadamma, K. C., Coutts, F. J., Mercer, T. H., Herman, J., Yirrel, J., Forbes, L., \& Van Der Linden, M. L. (2009). Effects of tuning of ankle foot orthoses-footwear combination using wedges on stance phase knee hyperextension in children with cerebral palsy preliminary results. Disabil Rehabil Assist Technol, 4(6), 406-413. doi:10.3109/17483100903104774

Jeans, K. A., Erdman, A. L., \& Karol, L. A. (2017). Plantar Pressures After Nonoperative Treatment for Clubfoot: Intermediate Follow-up at Age 5 Years. J Pediatr Orthop, 37(1), 53-58. doi:10.1097/BPO.0000000000000589

Kalampokas, E., Kalampokas, T., Sofoudis, C., Deligeoroglou, E., \& Botsis, D. (2012). Diagnosing arthrogryposis multiplex congenita: a review. ISRN Obstet Gynecol, 2012, 264918. doi:10.5402/2012/264918

Kimber, E., Tajsharghi, H., Kroksmark, A. K., Oldfors, A., \& Tulinius, M. (2012). Distal arthrogryposis: clinical and genetic findings. Acta Paediatr, 101(8), 877-887. doi:10.1111/j.1651-2227.2012.02708.x

Lowry, R. B., Sibbald, B., Bedard, T., \& Hall, J. G. (2010). Prevalence of multiple congenital contractures including arthrogryposis multiplex congenita in Alberta, Canada, and a strategy for classification and coding. Birth Defects Res A Clin Mol Teratol, 88(12), 1057-1061. doi:10.1002/bdra.20738

Nouraei, H., Sawatzky, B., MacGillivray, M., \& Hall, J. (2017). Long-term functional and mobility outcomes for individuals with arthrogryposis multiplex congenita. Am J Med Genet A, 173(5), 1270-1278. doi:10.1002/ajmg.a.38169

Obeidat, M. M., Audat, Z., \& Khriesat, W. (2012). Short-term functional outcome in children with arthrogryposis multiplex congenita after multiple surgeries at an early age. $J$ Multidiscip Healthc, 5, 195-200. doi:10.2147/JMDH.S31660 
Palmer, P. M., MacEwen, G. D., Bowen, J. R., \& Mathews, P. A. (1985). Passive motion therapy for infants with arthrogryposis. Clin Orthop Relat Res(194), 54-59.

Sells, J. M., Jaffe, K. M., \& Hall, J. G. (1996). Amyoplasia, the Most Common Type of Arthrogryposis: The Potential for Good Outcome. 97(2), 225-231.

Spencer, H. T., Bowen, R. E., Caputo, K., Green, T. A., \& Lawrence, J. F. (2010). Bone mineral density and functional measures in patients with arthrogryposis. J Pediatr Orthop, 30(5), 514-518. doi:10.1097/BPO.0b013e3181df6185

Staheli, L. T., Hall, J. G., Jaffe, K. M., \& Paholke, D. O. (1999). Arthrogryposis: A Text Atlas. $J$ Pediatr Orthop, 19(1), 138.

Toydemir, R. M., \& Bamshad, M. J. (2009). Sheldon-Hall syndrome. Orphanet J Rare Dis, 4, 11. doi:10.1186/1750-1172-4-11

van Bosse, H. J. P., Ponten, E., Wada, A., Agranovich, O. E., Kowalczyk, B., Lebel, E., . . . Durgut, F. (2017). Treatment of the Lower Extremity Contracture/Deformities. J Pediatr Orthop, 37 Suppl 1, S16-S23. doi:10.1097/BPO.0000000000001005

Veilleux, L. N., \& Rauch, F. (2017). Muscle-Bone Interactions in Pediatric Bone Diseases. Curr Osteoporos Rep, 15(5), 425-432. doi:10.1007/s11914-017-0396-6

Wren, T. A., Otsuka, N. Y., Bowen, R. E., Scaduto, A. A., Chan, L. S., Dennis, S. W., .. . Kay, R. M. (2013). Outcomes of lower extremity orthopedic surgery in ambulatory children with cerebral palsy with and without gait analysis: results of a randomized controlled trial. Gait Posture, 38(2), 236-241. doi:10.1016/j.gaitpost.2012.11.018

Zlotolow, D. A. (2017). Arthrogryposis. In S. W. Wolfe, R. N. Hotchkiss, W. C. Pederson, S. H. Kozin, M. S. Cohen, \& D. P. Green (Eds.), Green's operative hand surgery ClinicalKey (Seventh edition. ed., Vol. 7, pp. 1365-1390). Philadelphia, PA: Elsevier.

Zlotolow, D. A., \& Kozin, S. H. (2012). Posterior elbow release and humeral osteotomy for patients with arthrogryposis. J Hand Surg Am, 37(5), 1078-1082. doi:10.1016/j.jhsa.2012.02.032

Zlotolow, D. A., \& Tiedeken, N. C. (2014). Reorientation osteotomy for the atypical clasp thumb in children with arthrogryposis. Tech Hand Up Extrem Surg, 18(4), 165-169. doi:10.1097/BTH.0000000000000059 


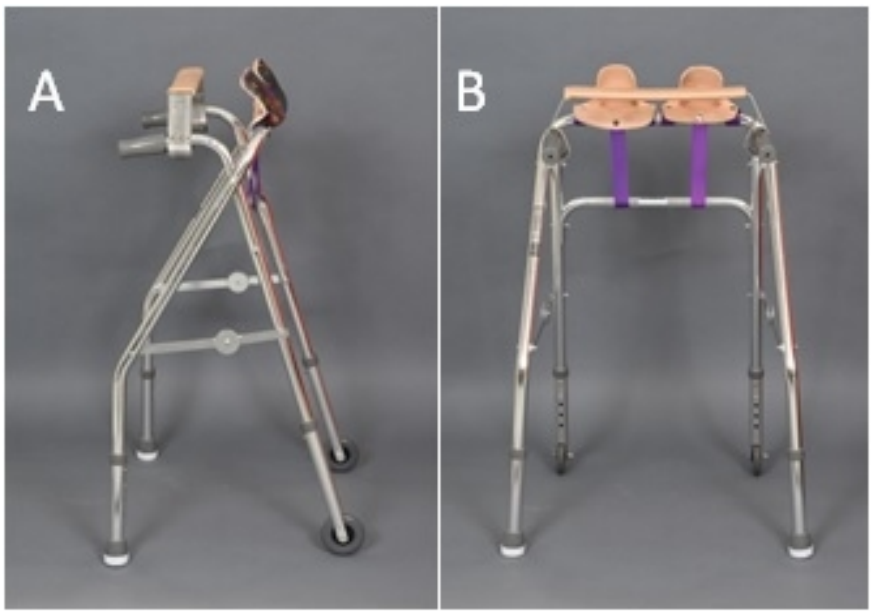

(A) Side view of wrist-hand orthoses on walker; (B) Frontal view of wrist-hand orthoses on walker $88 \times 61 \mathrm{~mm}(96 \times 96 \mathrm{DPI})$ 


$$
63 \times 89 \mathrm{~mm}(96 \times 96 \mathrm{DPI})
$$




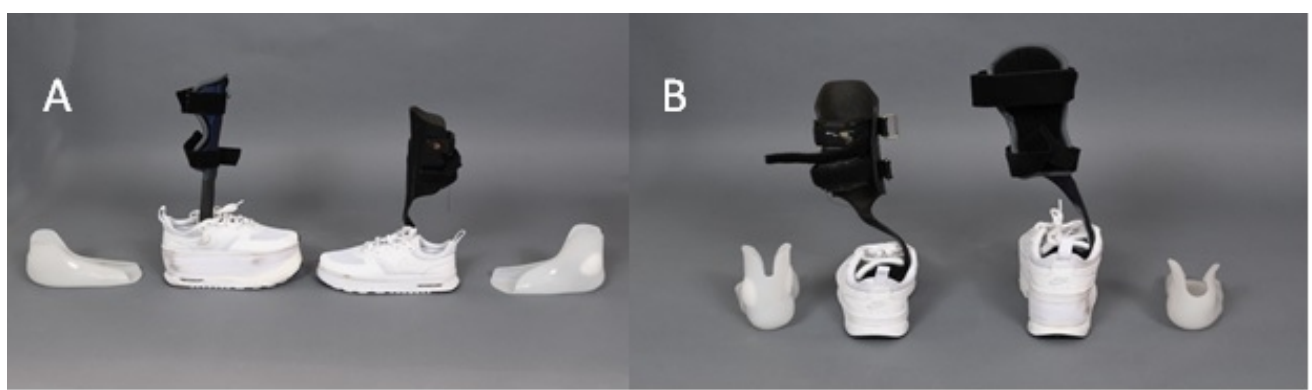

(A) Side view of Carbon Fiber Ankle Foot Orthoses (CF-AFO) with different heel lift shoes; (B) Posterior view of Carbon Fiber Ankle Foot Orthoses (CF-AFO) with different heel lift shoes

$164 \times 47 \mathrm{~mm}(96 \times 96 \mathrm{DPI})$ 